\title{
Auditorías de cumplimiento en los programas de fondos federales de los municipios de Puerto Rico
}

\author{
Dennis M. López / Dennis.Lopez@utsa.edu \\ University of Texas at San Antonio
}

\section{RESUMEN:}

Los municipios del Estado Libre Asociado de Puerto Rico son elegibles para recibir fondos del gobierno federal de los Estados Unidos. Este estudio presenta una discusión descriptiva de los fondos federales recibidos por estos municipios durante los años fiscales 2005 al 2009. También se discuten los hallazgos de las auditorías realizadas en conexión a estos fondos. Los resultados revelan que los municipios en la muestra recibieron un promedio de $\$ 8.2$ millones al año en fondos federales. El municipio de la ciudad capital de San Juan recibió un promedio de $\$ 126.5$ millones, convirtiéndolo en el mayor beneficiario de estos fondos durante el periodo de estudio. Los resultados también indican que 72.99 por ciento de las auditorías reveló deficiencias reportables y 31.02 por ciento de estas reveló deficiencias significativas. Además, los auditores independientes emitieron opiniones con salvedades para un 33.69 por ciento de las auditorías realizadas. Finalmente, 53.15 por ciento del total de los fondos auditados proviene de programas con hallazgos de auditoría.

Palabras clave: auditorías gubernamentales, Circular A-133, fondos federales, hallazgos de auditoría

\section{ABSTRACT:}

The municipalities of the Commonwealth of Puerto Rico are eligible to receive funds from the federal government of the United States. This study presents a descriptive discussion of the federal funding received by these municipalities during fiscal years 2005 to 2009. The findings of the audits performed in connection with these funds are also discussed. The results indicate that the municipalities in the sample received an average of $\$ 8.2$ million a year in federal funding. The municipality of San Juan, the capital city of Puerto Rico, received an average of $\$ 126.5$ million a year and was largest recipient of federal funds during the sample period. The results also indicate that 72.99 percent of the audits disclosed reportable conditions and 31.02 percent disclosed material weaknesses. In addition, auditors issued a qualified opinion report on 33.69 percent of the conducted audits. Lastly, 53.15 percent of all audited funds are associated with programs that disclosed audit findings.

Keywords: governmental audits, Circular A-133, federal funds, audit findings 


\section{INTRODUCCIÓN}

Las agencias federales del gobierno de los Estados Unidos auspician cientos de diversos programas cada año dirigidos al beneficio público y de la comunidad en general. Los fondos desembolsados por estos programas los reciben típicamente organizaciones gubernamentales a nivel estatal y local, entidades sin fines de lucro, entidades públicas, ciertas entidades privadas, grupos comunitarios e individuos particulares. Los municipios del Estado Libre Asociado de Puerto Rico son elegibles para recibir este tipo de fondos. Este estudio presenta una discusión descriptiva de los fondos federales recibidos por estos municipios. También se discuten los hallazgos de las auditorías de cumplimiento realizadas en conexión a estos fondos.

La Oficina de Gerencia y Presupuesto de los Estados Unidos requiere que toda entidad que reciba más de $\$ 500,000$ en fondos federales durante un año fiscal sea sometida a los requerimientos de auditoría y de control interno de la Circular A-133 (SAA, 1984; OMB, 2003). Las auditorías requeridas por la Circular A-133 conllevan ciertos requisitos particulares y pertenecen a la clasificación de auditorías de cumplimento. En un pasado, la gestión de auditoría de los programas de fondos federales era sumamente ineficiente y el proceso dependía grandemente de las estipulaciones individuales de cada programa. Como resultado, algunas entidades beneficiarias de estos fondos eran sobre auditadas, mientras que otras no recibían ningún tipo de escrutinio fiscal. La Ley de Auditorías Sencillas del 1984, mejor conocida en inglés como el "Single Audit Act", fue diseñada con el propósito de mejorar la consistencia y el alcance de las auditorías de estos programas.

A pesar de la magnitud de los fondos recibidos por las entidades beneficiarias de los programas de fondos federales, las auditorías de cumplimiento de la Circular A-133 continuamente revelan problemas de malversación de fondos, violaciones a las estipulaciones de de los programas y costos cuestionables. ${ }^{1}$ La Oficina del Contralor

${ }^{1}$ La Circular A-133 establece que los costos cuestionables son costos relacionados a transacciones en incumplimiento de la ley o en violación a las 
General de los Estados Unidos (GAO) ha emitido varios informes indicando que las agencias administradoras y los beneficiarios de estos programas no hacen lo suficiente para corregir los hallazgos de las auditorías de la Circular A-133 (GAO, 2002). En deterioro de la situación, el GAO y la literatura académica han documentado evidencia de deficiencias significativas en la calidad de las auditorías realizadas a los programas (GAO, 2007; López y Peters, 2010).

Los resultados de este estudio revelan que los municipios de Puerto Rico recibieron un promedio de $\$ 8.2$ millones al año en fondos federales durante el periodo cubierto por los años fiscales 2005 al 2009. El municipio de la ciudad capital de San Juan recibió un promedio de $\$ 126.5$ millones, convirtiéndolo en el mayor beneficiario de fondos federales en Puerto Rico durante el periodo de estudio. Los resultados también indican que 72.99 por ciento de las auditorías revelaron deficiencias reportables y 31.02 por ciento de estas revelaron deficiencias significativas. ${ }^{2}$ Además, los auditores independientes emitieron opiniones con salvedades para 33.69 por ciento de las auditorías realizadas. Finalmente, 53.15 por ciento del total de los fondos auditados provienen de programas con hallazgos de auditoría. Es decir, más de la mitad de los fondos federales recibidos por los municipios están relacionados con hallazgos que surgen como resultado de algún tipo de incumplimiento de la ley, violaciones a las estipulaciones de los programas, costos cuestionables, fraude o deficiencias significativas en el diseño y manejo de los sistemas de control interno, entre otros.

estipulaciones de los programas; costos para los cuales no existe documentación suficiente o adecuada al momento de la auditoría; o costos excesivos e irrazonables.

${ }^{2}$ Las Normas de Auditoría Generalmente Aceptadas, mejor conocidas por sus siglas en inglés como "GAAS", definen el concepto de deficiencias reportables como fallas en el sistema de control interno las cuales pudieran afectar la integridad de los informes financieros. Aquellas fallas con una mayor probabilidad de afectar la integridad de los informes son clasificadas como deficiencias significativas. En términos específicos a las auditorías examinadas en este estudio, estas deficiencias de control interno pudieran afectar la capacidad de los municipios de utilizar los fondos recibidos de acuerdo a la ley y las estipulaciones de los programas. 
El resto de este estudio está organizado de la siguiente manera. La segunda sección presenta una revisión de literatura. La tercera sección explica los métodos de investigación utilizados, mientras que la cuarta sección ofrece una discusión de los hallazgos. La quinta y última sección presenta las conclusiones y recomendaciones.

\section{REVISIÓn DE LITERATURA}

La Ley de Auditorías Sencillas fue promulgada en el 1984. Esta ley requiere que las entidades beneficiaras de fondos federales sean sujetas a los requerimientos de auditoría de la Circular A-133. En un pasado, la Circular A-133 requería que las entidades beneficiarias que recibieran más de $\$ 100,000$ en fondos federales fueran sujetas a una auditoría sencilla (SAA, 1984). En 1996 el requisito mínimo para estas auditorías fue incrementado a $\$ 300,000$ en fondos federales recibidos y luego a $\$ 500,000$ para las auditorías de años fiscales terminados a partir del 31 de diciembre del 2003. Antes de la creación de la Ley de Auditorías Sencillas, la función de fiscalización de los programas de fondos federales estaba enfocada en los programas sostenidos por estos fondos y no en las entidades beneficiarias de estos. Como resultado, algunas entidades eran sobre auditadas, mientras que otras no recibían ningún tipo de escrutinio fiscal.

Uno de los mayores beneficios de la Ley de Auditorías Sencillas es que esta requiere que toda entidad beneficiaria de los programas de fondos federales sea tratada como una entidad fiscal individual, independientemente de las distintas fuentes de los fondos recibidos por esta. La Ley también tiene como objetivo aumentar la consistencia y el alcance de las auditorías al requerir la preparación de informes de cumplimento con las regulaciones aplicables e informes de los hallazgos de deficiencias en los controles internos (Raman y Wilson, 1992; Wilson et al., 2007). Los requisitos específicos de los informes de auditoría y la divulgación de hallazgos de la Ley de Auditorías Sencillas están delineados en la Circular A-133 de la Oficina de Gerencia y Presupuesto (OMB, 2003).

En el 2002 el GAO emitió una serie de informes indicando que ciertas agencias del gobierno federal no estaban tomando las acciones pertinentes para asegurar que los hallazgos de las auditorías de 
la Circular A-133 no fueran pasados por alto (GAO 2002a, 2002b, 2002c). En términos generales, el GAO indica que muchas de las agencias administradoras de los programas tienen procedimientos establecidos para asegurar la distribución adecuada de los informes de auditoría. Sin embargo, la agencia también concluye que los administradores de los programas no están tomando las acciones necesarias para asegurar que las entidades beneficiarias de los fondos corrijan los hallazgos de los auditores independientes (GAO, 2002b). En deterioro de la situación, en el 2007 el GAO emite un informe indicando que existen problemas persistentes en la calidad y el alcance de las auditorías de la Circular A-133 (GAO, 2007). El informe, el cual está basado en los hallazgos de un estudio preparado por la Oficina de Gerencia y Presupuesto, indica que más de la mitad de las auditorías revisadas contienen deficiencias crasas. El GAO concluye en el informe que las auditorías revisadas deben verse como auditorías con limitaciones de alcance o simplemente como auditorías de calidad inaceptable.

A pesar de los continuos señalamientos del GAO y otras entidades relacionadas, la literatura académica en el área de las auditorías gubernamentales y de entidades sin fines de lucro es relativamente escasa. Además, muchos de los estudios en el área presentan resultados contradictorios o inconclusos. Por ejemplo, un estudio reciente analiza los factores determinantes de los hallazgos de problemas de control interno en entidades sin fines de lucro y concluye que los auditores independientes de las firmas internacionales de contadores públicos autorizados solo se relacionan con clientes que tienen un buen sistema de control interno (Petrovits et al., 2011). Por lo tanto, las auditorías realizadas por estas firmas deben presentar una menor cantidad de hallazgos. Keating et al. (2005) también utilizan una muestra de auditorías de la Circular A-133 y documentan resultados similares.

Por otro lado, Ashbaugh et al. (2007) establecen que las auditorías ejecutadas por las firmas de contadores públicos autorizados con una posición dominante en el mercado deben estar asociadas con una mayor probabilidad de hallazgos, ya que estas firmas poseen un mayor grado de experiencia y conocimiento especializado. Utilizando una muestra de auditorías de la Circular A-133, López y 
Peters (2010) encuentran que las auditorías realizadas por las firmas de contadores públicos autorizados tienen una mayor probabilidad de revelar hallazgos de control interno que las auditorías realizadas por las agencias del gobierno. Los investigadores concluyen que las discrepancias observadas surgen como parte de las diferencias en los factores de riesgo asociados con los distintos tipos de auditores independientes en el estudio. López y Peters (2010) también presentan evidencia de una mayor incidencia de hallazgos en las auditorías llevadas a cabo por las firmas de contadores públicos autorizados de mayor tamaño.

\section{Metodología}

Los datos en este estudio provienen del repositorio de datos de las auditorías de la Circular A-133 de la Oficina del Censo de los Estados Unidos. Este repositorio, mejor conocido en inglés como el "Federal Audit Clearinghouse", contiene un archivo completo de los informes de las auditorías de la Circular A-133 y de los fondos federales relacionados a estas. La muestra consiste de los informes de las auditorías sencillas de los 78 municipios de Puerto Rico para los años fiscales 2005 al 2009. Los informes fueron identificados mediante una búsqueda de palabras clave con los nombres de los municipios. Dada la cantidad de años cubiertos por la muestra, hay un posible máximo de 5 observaciones en cada sección transversal de datos. Sin embargo, algunas de estas secciones contienen solo 3 o 4 observaciones, ya que al momento de tomar la muestra el informe de auditoría del municipio aún permanecía incompleto o el municipio recibió menos de $\$ 500,000$ en fondos federales durante el año, eximiéndolo de los requisitos de la Ley de Auditorías Sencillas.

Los procedimientos delineados en el párrafo anterior identifican un total de 374 observaciones de secciones transversales. Todos los municipios de Puerto Rico están representados en la muestra y los datos son comparables con los datos utilizados en otros estudios basados en las auditorías de la Circular A-133 (Jakubowski, 1995; McLelland y Giroux, 2000; Johnson et al., 2002; Payne y Jensen, 2002; Giroux y McLelland, 2003; López y Peters, 2010). Cabe resaltar que una cantidad significativa de estos estudios analiza las auditorías de 
los condados, ciudades, municipalidades y gobiernos locales de los Estados Unidos. Aunque el tamaño de estas entidades puede ser extenso, las operaciones de estas son análogas a las de los municipios de Puerto Rico. Finalmente, y de acuerdo a los informes de auditoría incluidos en la muestra, los fondos federales recibidos por los municipios provienen de programas auspiciados por el Departamento de Agricultura, Departamento de la Vivienda, Departamento de Justicia, Departamento del Trabajo, Departamento de Transportación, Departamento de Salud y el Departamento de Seguridad Nacional, entre otras agencias.

Se presentan dos análisis de estadísticas descriptivas en este estudio. El primer análisis está basado en los fondos federales recibidos por los municipios durante el periodo de la muestra. El segundo análisis está basado en los hallazgos de las auditorías de la Circular A-133 realizadas a los municipios. En este segundo análisis se destaca el por ciento de auditorías con deficiencias reportables, deficiencias significativas, costos cuestionables y opiniones con salvedades. También se presenta el por ciento de fondos auditados provenientes de programas con hallazgos de auditoría.

\section{Discusı́ón}

La Tabla 1 contiene estadísticas descriptivas de los fondos federales recibidos por los municipios de Puerto Rico. El Panel A de esta tabla presenta información detallada acerca de los municipios principales de la isla, mientras que el Panel B presenta un sumario por áreas principales. Como presentado en el Panel A, el municipio de la ciudad capital de San Juan recibió un promedio de $\$ 126.5$ millones, convirtiéndolo en el mayor beneficiario de fondos de programas federales en Puerto Rico durante el periodo cubierto por la muestra. Con un promedio de $\$ 53.5$ millones, el municipio de Bayamón fue el segundo mayor beneficiario de estos fondos. Por otro lado, el municipio de Caguas recibió un promedio de \$29.1 millones, lo cual lo convierte en el mayor beneficiario de fondos federales entre los municipios localizados en áreas limítrofes al área metropolitana de San Juan. Finalmente, el municipio de Ponce recibió un promedio de $\$ 38.2$ millones, convirtiéndolo en el mayor beneficiario de fondos 
federales entre los municipios localizados en áreas metropolitanas secundarias al área metropolitana de San Juan.

El sumario por áreas principales de Puerto Rico, presentado en el Panel B de esta tabla, indica que los municipios recibieron un promedio de $\$ 8.2$ millones durante el periodo cubierto por la muestra. Esto representa un total de $\$ 3.1$ billones en fondos recibidos o un promedio de $\$ 610.4$ millones por año. Con un promedio de $\$ 40.0$ millones y $\$ 22.8$ millones, los municipios del área metropolitana de San Juan y de otras áreas metropolitanas secundarias recibieron respectivamente la mayor cantidad de fondos. Los datos en el Panel B también indican que los fondos recibidos por los municipios fluctúan entre $\$ 0.5$ millones y $\$ 134.6$ millones. Estas cantidades pertenecen a los municipios de Culebra en el 2006 y San Juan en el 2005, respectivamente.

La Tabla 2 presenta los hallazgos de las auditorías de la Circular A-133. El Panel A de esta tabla deja ver que la gran mayoría de las auditorías de los municipios del área metropolitana de San Juan reveló deficiencias reportables. Por ejemplo, todas las auditorías realizadas a los municipios de Carolina, Cataño, Guaynabo y San Juan durante el periodo de la muestra revelaron dichas deficiencias. Un patrón similar se puede observar en las auditorías de los municipios en áreas limítrofes al área metropolitana de San Juan y las auditorías de los municipios en áreas metropolitanas secundarias. En este caso, todas las auditorías realizadas a los municipios de Aguas Buenas, Arecibo, Canóvanas, Mayagüez, Ponce y Toa Baja revelaron deficiencias reportables. Además, al menos un 40 por ciento de las auditorías realizadas al resto de los municipios presentados en el Panel A también reveló este tipo de deficiencias.

La situación de las auditorías con deficiencias significativas, costos cuestionables u opiniones con salvedades es similar. Por ejemplo, todas las auditorías realizadas a los municipios de Arecibo, Canóvanas, Cataño, Ponce y San Juan revelaron deficiencias significativas; todas las auditorías realizadas al municipio de Ponce revelaron costos cuestionables; y todas las auditorías realizadas a los municipios de Ponce, San Juan y Toa Baja recibieron informes de opinión con salvedades. La última columna de este panel presenta la proporción de fondos federales auditados provenientes de programas con 
hallazgos de auditoría. Estos hallazgos surgen como resultado de incumplimiento de la ley, violaciones a las estipulaciones de los programas, costos cuestionables, fraude o deficiencias significativas en el diseño y manejo de los sistemas de control interno, entre otros. Los datos revelan que al menos un 80 por ciento de los fondos federales recibidos por los municipios de Arecibo, Canóvanas, Cataño, Ponce y San Juan están asociados a programas con estos tipos de hallazgos.

El sumario por áreas principales de Puerto Rico, presentado en el Panel B de esta tabla, indica que aproximadamente 72.99 por ciento de las auditorías reveló deficiencias reportables y 31.02 por ciento de estas reveló deficiencias significativas. Con un promedio de 86.21 por ciento y 85.00 por ciento, los municipios del área metropolitana de San Juan y de otras áreas metropolitanas secundarias respectivamente presentan las mayores proporciones de auditorías con deficiencias reportables. El promedio de auditorías con deficiencias significativas es de 44.83 por ciento y 65.00 por ciento para los municipios del área metropolitana de San Juan y de otras áreas metropolitanas secundarias, respectivamente. Además, los auditores independientes emitieron opiniones con salvedades para aproximadamente 33.69 por ciento de las auditorías realizadas. Cabe señalar que el promedio de opiniones con salvedades recibidas por los municipios del área metropolitana de San Juan es de 58.62 por ciento. Los resultados también revelan que un 53.15 por ciento del total de fondos auditados provienen de programas con hallazgos de auditoría. Los programas de los municipios del área metropolitana de San Juan y de otras áreas metropolitanas secundarias están asociados con las mayores proporciones de fondos federales provenientes de programas con hallazgos.

\section{ConCLUSIONES y RECOMENDACIONES}

El gobierno federal de los Estados Unidos desembolsa billones de dólares todos los años para el beneficio de diferentes programas. Toda entidad beneficiaria que reciba más de $\$ 500,000$ en fondos federales está obligada a cumplir con los requisitos de auditoría de la Circular A-133 (OMB, 2003). A pesar de la magnitud de los 
fondos federales desembolsados y de los esfuerzos del gobierno federal para mejorar la administración de estos programas, existe evidencia que sugiere que las agencias administradoras y los beneficiarios de estos programas no están haciendo lo suficiente para corregir los hallazgos de las auditorías de la Circular A-133 (GAO, 2002b). En deterioro de la situación, existe evidencia de deficiencias significativas en la calidad de las auditorías realizadas a los programas (GAO, 2007; López y Peters, 2010).

Este estudio presenta información descriptiva acerca de los fondos recibidos por los programas de fondos federales de los municipios de Puerto Rico. En congruencia con los comentarios del GAO, los municipios incluidos en la muestra aparentan no corregir los hallazgos de las auditorías de la Circular A-133. Los resultados demuestran que una gran mayoría de las auditorías realizadas a estos municipios reveló deficiencias reportables, deficiencias significativas, costos cuestionables y otros hallazgos de auditoría. Más importante aún es el hecho de que para varios de los municipios en cuestión, particularmente aquellos localizados en el área metropolitana de San Juan y en otras áreas metropolitanas secundarias, los hallazgos aparentan ser de tipo recurrente. Por ejemplo, todas las auditorías realizadas a los municipios de Aguas Buenas, Arecibo, Canóvanas, Carolina, Cataño, Guaynabo, Mayagüez, Ponce, San Juan y Toa Baja revelaron debilidades reportables durante los cinco años cubiertos por la muestra. Asimismo, todas las auditorías de los municipios de Arecibo, Canóvanas, Cataño, Ponce y San Juan también revelaron debilidades significativas durante dicho periodo.

Dada la magnitud de los fondos federales recibidos, un mayor escrutinio fiscal y mejorías significativas a los sistemas de control interno aparentan ser de primer orden para los municipios de Puerto Rico. Aunque este estudio documenta una alta incidencia de hallazgos en las auditorías realizadas, la situación puede ser mejorada con la introducción de algunas medidas básicas. Por ejemplo, los municipios se beneficiarían grandemente de la creación e implementación de un plan formal para la corrección de los hallazgos de los auditores. Es de suma importancia que los municipios se aseguren de corregir todas las fallas de control interno reveladas, ya que esto puede afectar su elegibilidad para nuevos programas en el futuro. También 
es importante que los informes de auditoría y los hallazgos de los auditores sean comunicados a todas las partes interesadas para que el proceso de mejoras sea más eficiente y efectivo.

Futuras investigaciones sobre este tema pudieran estar orientadas a evaluar si ciertos factores específicos a las operaciones de los municipios de Puerto Rico, como la designación de municipio autónomo, tienen algún tipo de impacto en la incidencia de los hallazgos de auditoría investigados en este estudio. La Ley de Municipios Autónomos del 1991 le otorga a los municipios de la isla un mayor grado de autonomía fiscal y de gobierno propio (LMAPR, 1991). Por lo tanto, es probable que los municipios que ya han recibido tal designación tengan operaciones más complejas y que sus sistemas de control interno sean distintos. Asimismo, el área de gobierno propio en el ámbito de las instituciones sin fines de lucro y de las entidades gubernamentales provee un terreno fértil para la investigación de factores que hasta este momento han sido mayormente investigados desde el punto de las grandes empresas. 


\section{Referencias}

Ashbaugh-Skaife, H., Collins, D. W., \& Kinney, W. R. (2007). The discovery and reporting of internal control deficiencies prior to SOX-mandated audits. Journal of Accounting and Economics, 44(1-2), 166-192.

Giroux, G., \& McLelland, A. J. (2003). Governance structures and accounting at large municipalities. Journal of Accounting and Public Policy, 22(3), 203-230.

Jakubowski, S. (1995). Reporting on the control structures of local government under the Single Audit Act of 1984. Public Budgeting and Finance, 15(1), 58-71.

Johnson, L. E., Davies, S. P., \& Freeman, R. J. (2002). The effect of seasonal variations in auditor workload on local government audit fees and audit delay. Journal of Accounting and Public Policy, 21(4-5), 395-422.

Keating, E. K., Fischer, M., Gordon, T. P., \& Greenlee, J. (2005). The Single Audit Act: How compliant are nonprofit organizations? Journal of Public Budgeting, Accounting and Financial Management, 17(3), 285-309.

López, D. M., \& Peters, G. F. (2010). Internal control reporting differences among public and governmental auditors: The case of city and county Circular A-133 audits. Journal of Accounting and Public Policy, 29(5), 481-502.

McLelland, A. J., \& Giroux, G. (2000). An empirical analysis of auditor report timing by large municipalities. Journal of Accounting and Public Policy, 19(3), 263-281.

Office of Management and Budget (OMB). (2003). Circular No. A-133: Audits of States, Local Governments, and Non-Profit Organizations. Washington, DC: Office of Management and Budget.

Payne, J. L., \& Jensen, K. L. (2002). An examination of municipal audit delay. Journal of Accounting and Public Policy 21(1), 1-29.

Petrovits, C., Shakespeare, C., \& Shih, A. (2011). The causes and consequences of internal control problems in nonprofit organizations. The Accounting Review (forthcoming). 
Raman, K. K., \& Wilson, E. R. (1992). An empirical investigation of the market for "single audit" services. Journal of Accounting and Public Policy, 11(4), 271-295.

Senado de Puerto Rico. (1991). Ley 81 de 30 de agosto de 1991, Ley de Municipios Autónomos de Puerto Rico (LMAPR). San Juan, PR: Senado de Puerto Rico.

The Single Audit Act (SAA), Pub. L. No. 98-502, S 98, Stat. 2327 (1984).

U.S. Government Accountability Office (GAO). (2002a). Survey of CFO Act agencies (GAO-02-376). Washington, DC: U.S. Government Accountability Office.

U.S. Government Accountability Office (GAO). (2002b). Single audit: Actions needed to ensure that findings are corrected (GAO-02-705). Washington, DC: U.S. Government Accountability Office.

U.S. Government Accountability Office (GAO). (2002c). Single audit: Single Audit Act effectiveness issues (GAO-02-877T). Washington, DC: U.S. Government Accountability Office.

U.S. Government Accountability Office (GAO). (2007). Single audit quality: Actions needed to address persistent audit quality problems (GAO-08-213T). Washington, DC: U.S. Government Accountability Office.

Wilson E. R., Kattelus S. C., \& Reck J. L. (2007). Accounting for governmental and nonprofit entities (14th ed.). New York, NY: McGrawHill Irwin. 


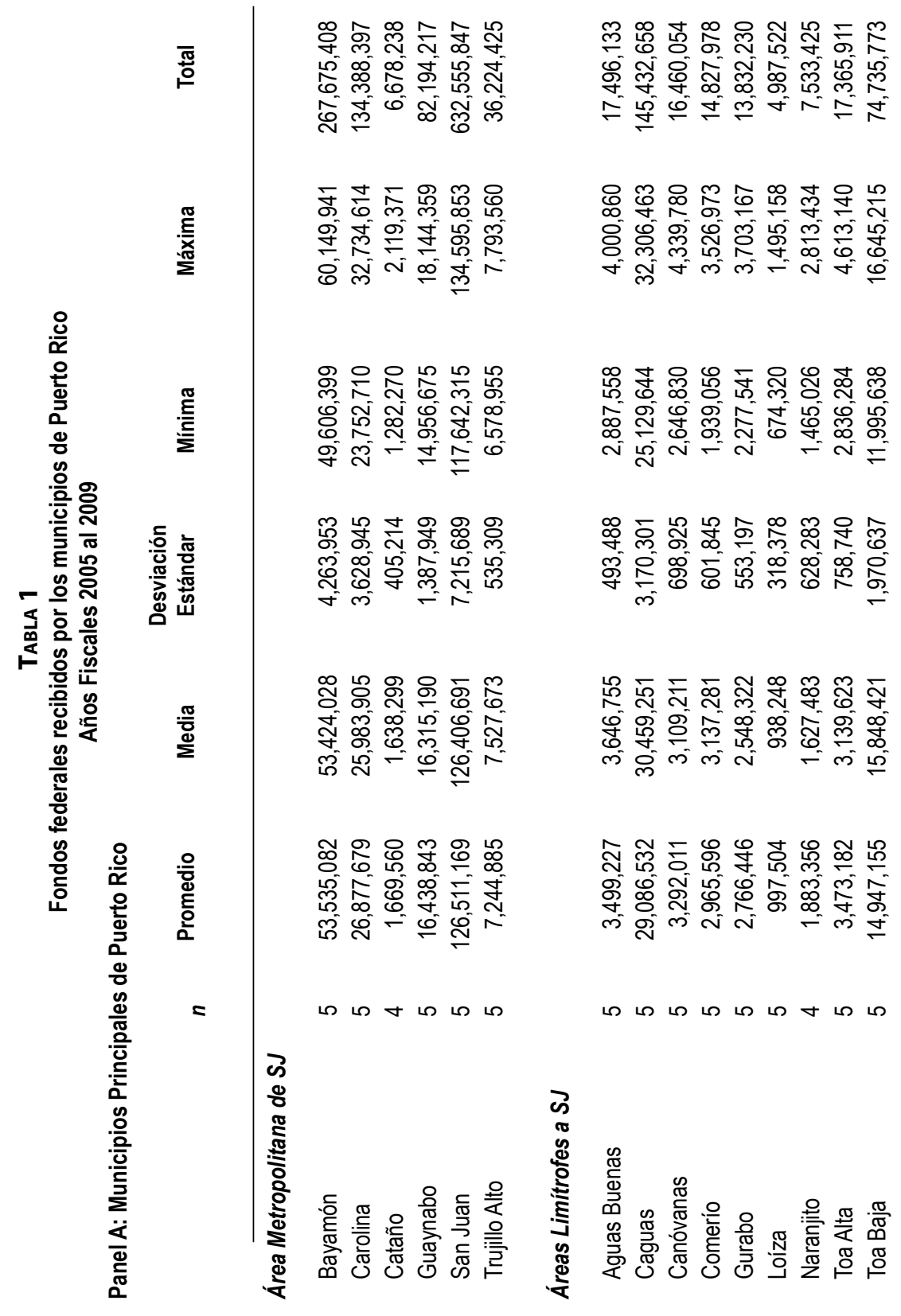




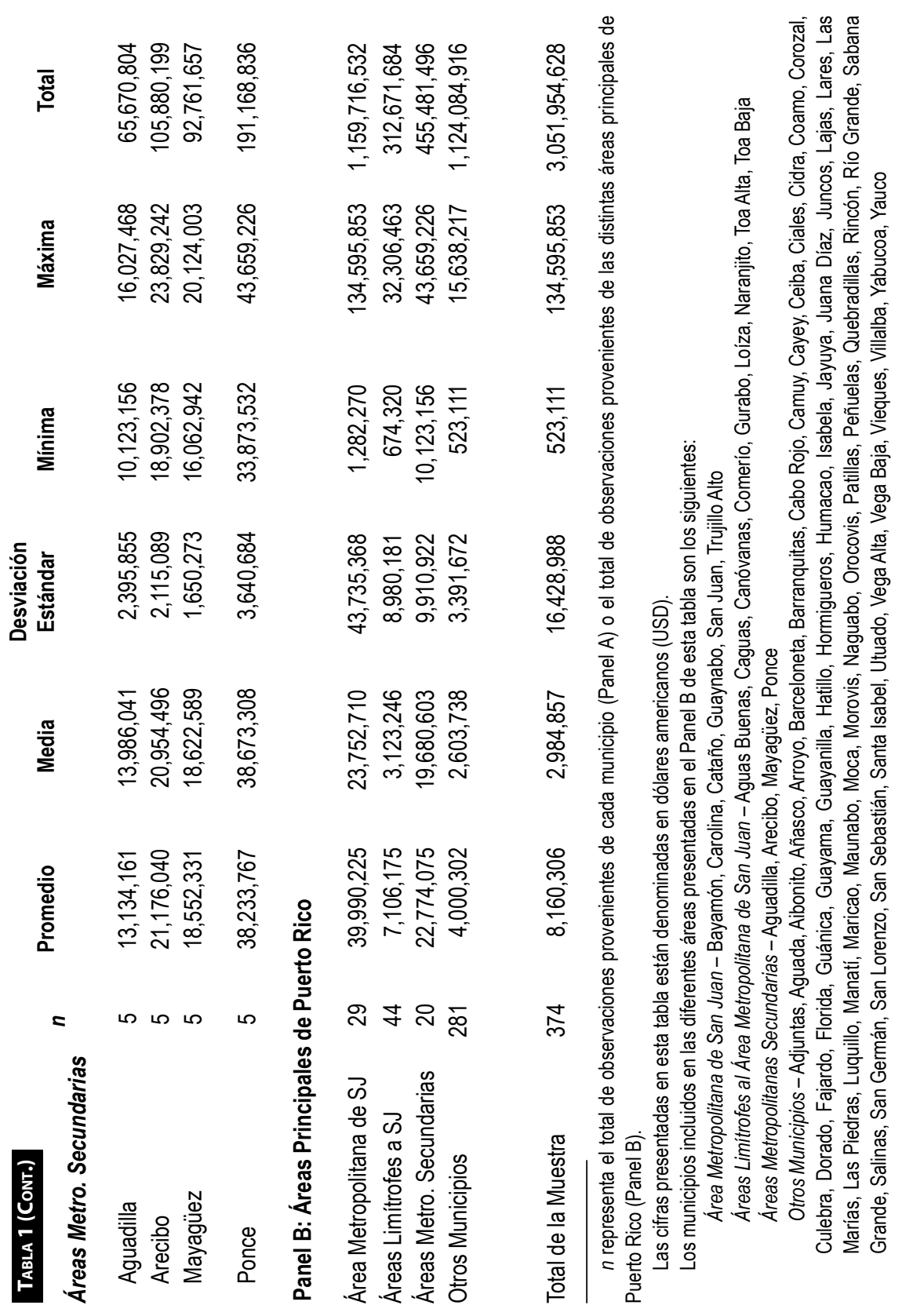




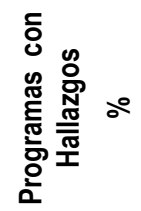

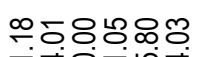

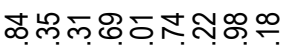

๒ூ유요

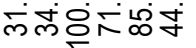

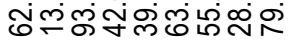

ம்ं்

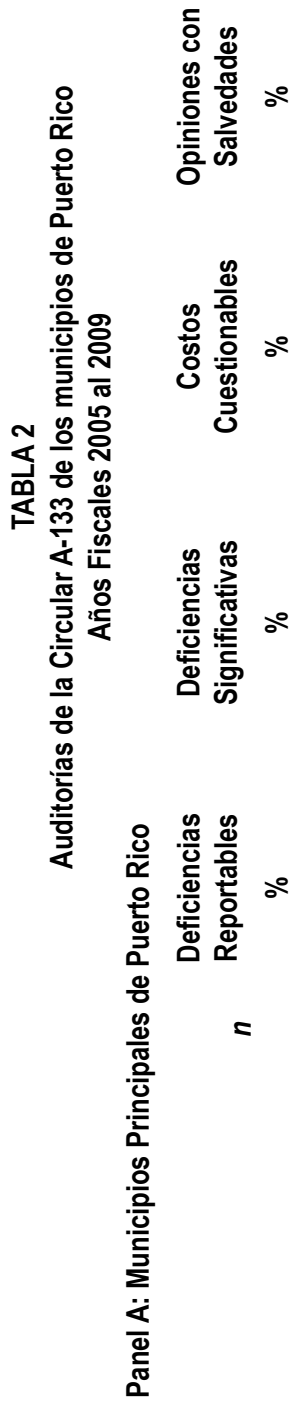

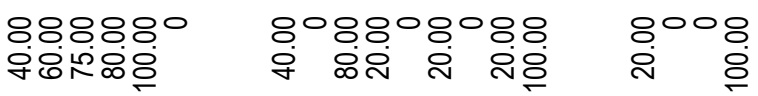

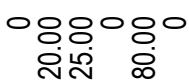

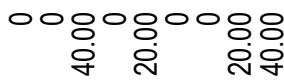

8888

뒤용

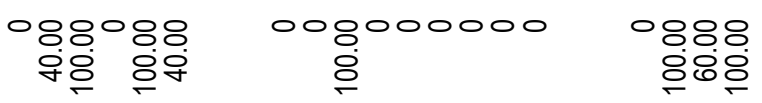

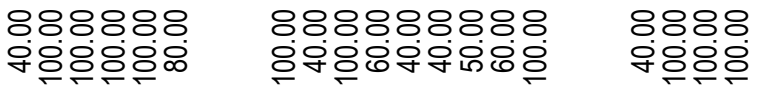

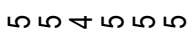

ما ما ף ما ما ما ما ما ما

ما ما ما ما

क

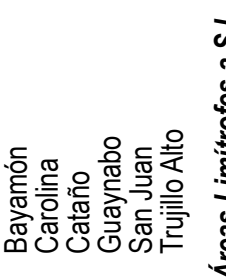

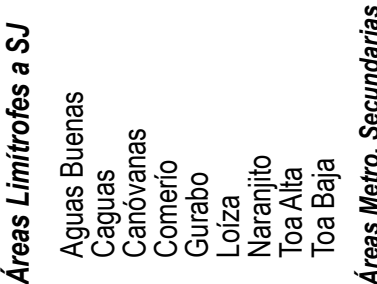

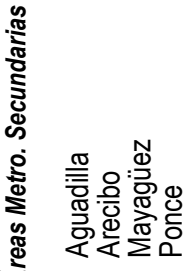



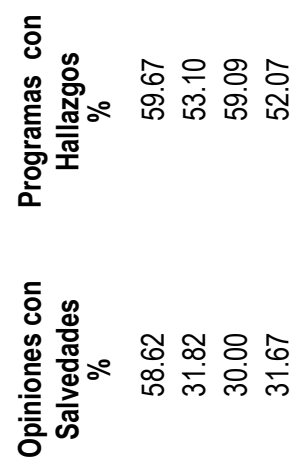

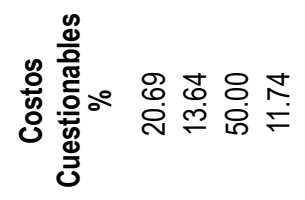

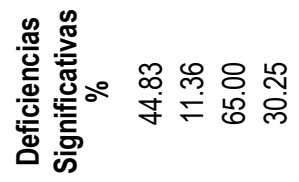

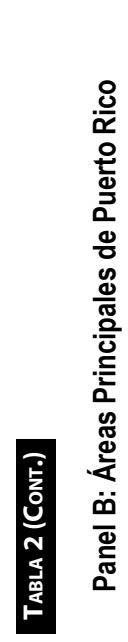

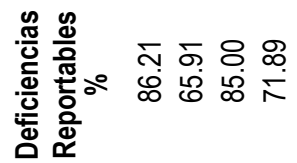
त्ञ त

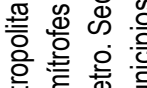

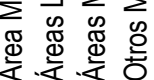

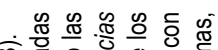

Фิ

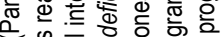

응 뜬 은 은응 은

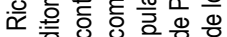

เ

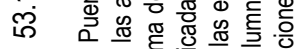

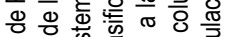

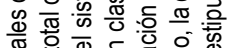

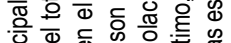

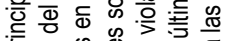

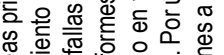

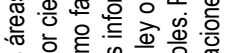

ஓ ๘

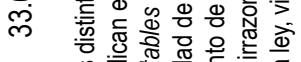

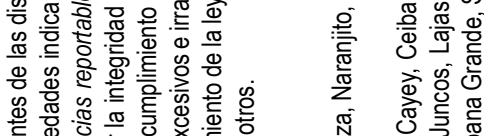

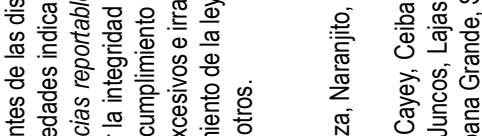

论

क ल

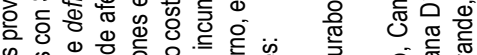

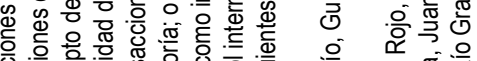

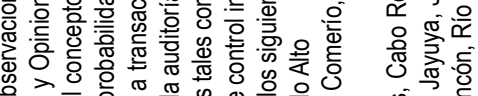

\&

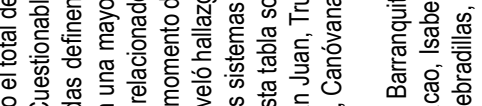

穴

ब ॠ

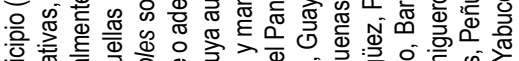

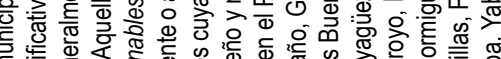

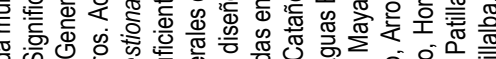

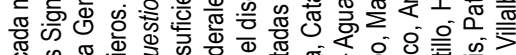

ᄋ)

N

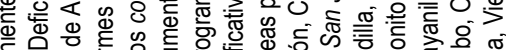

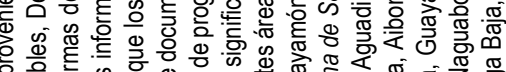

远 은

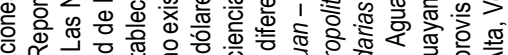

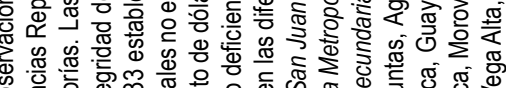

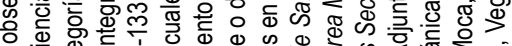

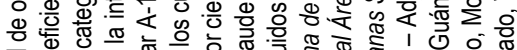
퓸 옹 ․ㅡㄹ 을
ब

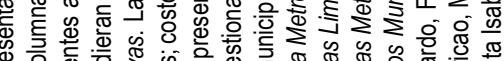

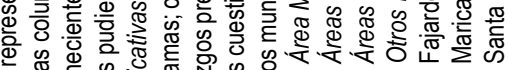

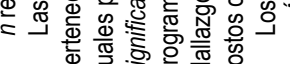


